\title{
LOCAL INVERSION OF PLANAR MAPS WITH NICE NONDIFFERENTIABILITY STRUCTURE
}

\author{
LAURA POGGIOLINI AND MARCO SPADINI
}

\section{INTRODUCTION}

Inspired by invertibility problems for $\mathrm{PC}^{1}$ maps (see e.g., [6]) that naturally arise in Optimal Control (see e.g., [11) we focus on the invertibility of continuous maps of the plane which are piecewise linear.

When the plane is pie-sliced in $n \leq 4$ parts (with nonempty interior and common vertex at the origin) our main result, Theorem 4.5 below, provides a sufficient condition for any map L, that is continuous and piecewise linear relatively to this slicing, to be invertible. Some examples show that the assumptions of the theorem cannot be relaxed too much. In particular, convexity of the slices cannot be dropped altogether when $n=4$ and, perhaps not surprisingly, this result cannot be plainly extended to a greater number of slices. This result is proved by a combination of linear algebra and topological arguments in which Theorems 4 and 5 of [9] (Theorems 2.3 and 2.4 below) play a crucial role. By contrast, an important tool of nonsmooth analysis, Clarke's Theorem [4, does not appear to be adequate for our purposes in the case $n=4$. We exhibit an explicit example that shows how this case cannot be treated completely by Clarke's Theorem.

Our results depend on the particularly nice nondifferentiability structure that we assume throughout. In fact example 2.1 in [6 shows that there exists a $\mathrm{PC}^{1}$ function with 4 selection functions (which does not have such structure) which is not locally invertible at the origin despite being Fréchet differentiable at 0 with invertible differential.

As stated above, our interest in the invertibility of $\mathrm{PC}^{1}$ maps stems from optimal control problems. Namely, if one considers a multiinput optimal control problem which is affine with respect to the control variable $u \in[-1,1]^{\mathrm{m}}$, then one cannot exclude the existence of bang-bang Pontryagin extremals. This gives rise to a $\mathrm{PC}^{1}$ maximized Hamiltonian flow. In order to prove the optimality of the given Pontryagin extrema via Hamiltonian methods, one needs to prove the invertibility of the projection of such flow on the state space (see [1] for an introduction to Hamiltonian methods in control and 2, 12 for specific applications to bang-bang Pontryagin extremals). In particular, as in 10, 11] we are interested in what happens when two control components switch simultaneously just once. In this case the "interesting" part of the above-mentioned projection is 2-dimensional. This justifies our interest into the invertibility of planar maps. Moreover, a double switch gives rise to the "nice" nondifferentiability structure we consider in this paper with at most $n=5$ pie-slices which reduce to 4 for the subsequent simple switches.

To the best of our knowledge, a comprehensive treatment of invertibility results in simple cases is not available in the literature. This has, perhaps, slowed down the study of bang-bang Pontryagin extremals with multiple switch behavior.

Some comments are in order concerning some of the illustrations included in this paper. Figures 1 , 2 and 4 represent the piecewise linear maps contained in Examples 4.6 4.7 and 4.14, respectively. In fact, they actually show the image of the unit circle $S^{1}$ under these maps. But, for the sake of clarity, we have altered 
the proportion between axes and, in order to enhance the view close to the origin, we logarithmically rescaled the radial distance from the origin. Notice that such transformations do not change the qualitative behavior of the maps (at least not the characteristics we are interested in).

\section{Preliminaries And NOtATion}

2.1. Some notions of nonsmooth analysis. Following 6 , a continuous function $\mathrm{f}: \mathrm{U} \subseteq \mathbb{R}^{s} \rightarrow \mathbb{R}^{\mathrm{m}}$ is a continuous selection of $\mathrm{C}^{1}$ functions if there exists a finite number of $C^{1}$ functions $f_{1}, \ldots, f_{\ell}$, of $U$ into $\mathbb{R}^{m}$ such that the active index set $\mathcal{I}:=\left\{i: f(x)=f_{i}(x)\right\}$ is nonempty for each $x \in \mathcal{U}$. The functions $f_{i}$ 's are called selection functions of $\mathrm{f}$. The function $\mathrm{f}$ is called a $\mathrm{PC}^{1}$ function if at every point $x \in \mathrm{U}$ there exists a neighborhood $\mathrm{V}$ such that the restriction of $f$ to $\mathrm{V}$ is a continuous selection of $\mathrm{C}^{1}$ functions.

A function $f: \mathbb{R}^{s} \rightarrow \mathbb{R}^{m}$ is said to be piecewise linear if it is a continuous selection of linear functions. We will actually focus on a much more restrictive class of piecewise linear functions namely in the case $\mathrm{m}=\mathrm{s}=2$.

Definition 2.1. A cone with nonempty interior $C$ and vertex at the origin of $\mathbb{R}^{k}$ is called a polyhedral cone if it is the intersection of a finite number of half-spaces.

Definition 2.2. We say that a continuous map $\mathrm{G}: \mathbb{R}^{\mathrm{k}} \rightarrow \mathbb{R}^{\mathrm{k}}$ is strongly piecewise linear (at 0 ) if there exist a decomposition $\mathrm{C}_{1}, \ldots, \mathrm{C}_{\mathrm{n}}$ of $\mathbb{R}^{\mathrm{k}}$ in closed polyhedral cones with nonempty interior and common vertex at the origin, and linear maps $\mathrm{L}_{1}, \ldots, \mathrm{L}_{\mathrm{n}}$ with

$$
\mathrm{G}(x)=\mathrm{L}_{\mathrm{i}} x, \quad x \in \mathrm{C}_{\mathrm{i}} .
$$

We also say that $\mathrm{G}$ is nondegenerate if $\operatorname{sign}\left(\operatorname{det} \mathrm{L}_{i}\right)$ is constant and nonzero for all $i=1, \ldots, n$.

Notice that if $G$ is a continuous strongly piecewise linear map as in Definition 2.2 above, then $L_{i} x=L_{j} x$ for any $x \in C_{i} \cap C_{j}$ and $i, j \in\{1, \ldots, n\}$. Moreover, $G$ is positively homogeneous.

In this paper we are concerned with the global invertibility of continuous nondegenerate strongly piecewise linear maps. In this regard the following simple observation is in order:

Lemma 2.1. Let $\mathrm{G}: \mathbb{R}^{\mathrm{k}} \rightarrow \mathbb{R}^{\mathrm{k}}$ be a continuous strongly piecewise linear map as in Definition 2.2, and let $\mathrm{U}$ be an open neighborhood of $0 \in \mathbb{R}^{\mathrm{k}}$. Assume that the restriction $\left.\mathrm{G}\right|_{\mathrm{U}}: \mathrm{U} \rightarrow \mathrm{G}(\mathrm{U})$ is invertible with continuous inverse, then $\mathrm{G}$ is globally invertible and its inverse is a continuous strongly piecewise linear map as well.

Proof. Let us first prove that $\mathrm{G}$ is injective. Let $x_{1}, \mathrm{x}_{2} \in \mathbb{R}^{\mathrm{k}}$ be such that $\mathrm{G}\left(\mathrm{x}_{1}\right)=$ $\mathrm{G}\left(x_{2}\right)$. Let $\rho>0$ be such that the sphere $S_{\rho}$ of radius $\rho$ and centered at the origin is contained in $\mathrm{U}$. Then

$$
G\left(\rho \frac{x_{1}}{\left\|x_{1}\right\|}\right)=G\left(\rho \frac{x_{2}}{\left\|x_{2}\right\|}\right) .
$$

Since, for $i=1,2, \rho x_{i} /\left\|x_{i}\right\| \in U$, we get $x_{1}=x_{2}$.

Let us now prove surjectivity by explicitly exhibiting the inverse. This will take care of the continuity too. Given $y \in \mathbb{R}^{k}$, define $H(y)$ as follows:

$$
H(y):=\frac{\|y\|}{\rho}\left(\left.G\right|_{u}\right)^{-1}\left(\rho \frac{y}{\|y\|}\right)
$$

where $\rho$ is as above. Clearly the above definition does not depend on the choice of $\rho$. The fact that $\mathrm{G}(\mathrm{H}(\mathrm{y}))=\mathrm{y}$ for any $\mathrm{y} \in \mathbb{R}^{k}$ is a straightforward computation. 
In this paper, we study the invertibility of continuous strongly piecewise linear maps. We will prove later (Proposition 4.1 below) that, if such a map is invertible, then it is necessarily nondegenerate. It is not difficult to see that the converse of this statement is not true (see for instance Examples 4.6 and 4.7 below). Our main concern will be finding simple sufficient conditions for the invertibility. Section 4 is devoted to this purpose. Before dealing with this problem, however, we need some preliminaries.

A classical notion which we need is that of Bouligand derivative. Let $\mathrm{U} \subseteq \mathbb{R}^{\mathrm{s}}$ be open and let $\mathrm{f}: \mathrm{U} \rightarrow \mathbb{R}^{\mathrm{m}}$ be locally Lipschitz. We say that $\mathrm{f}$ is Bouligand differentiable at $x_{0} \in \mathrm{U}$ if there exists a positively homogeneous function, $f^{\prime}\left(x_{0}, \cdot\right): \mathbb{R}^{s} \rightarrow$ $\mathbb{R}^{\mathrm{m}}$ with the property that

$$
\lim _{x \rightarrow x_{0}} \frac{\left\|f(x)-f\left(x_{0}\right)-f^{\prime}\left(x_{0}, x-x_{0}\right)\right\|}{\left\|x-x_{0}\right\|}=0 .
$$

This uniquely determined function $f^{\prime}\left(x_{0}, \cdot\right)$ is called the Bouligand derivative of $f$ at $x_{0}$. An important fact proved by Kuntz/Scholtes [6] is the following:

Proposition 2.2 (Prop. 2.1 in [6]). Let $\mathrm{U} \subseteq \mathbb{R}^{s}$ be an open set. A $\mathrm{PC}^{1}$ function $\mathrm{f}: \mathrm{U} \rightarrow \mathbb{R}^{\mathrm{m}}$ is locally Lipschitz and, at every $\mathrm{x}_{0} \in \mathrm{U}$, has a piecewise linear Bouligand derivative $\mathrm{f}^{\prime}\left(\mathrm{x}_{0}, \cdot\right)$ which is a continuous selection of the Fréchet derivatives of the selection functions of $\mathrm{f}$ at $\mathrm{x}_{0}$.

Following 9 we consider a generalization of the notion of Jacobian matrix $\nabla f(x)$ of a function $f: \mathbb{R}^{k} \rightarrow \mathbb{R}^{k}$ at a Fréchet differentiability point $x$. Let $f: \mathbb{R}^{k} \rightarrow \mathbb{R}^{k}$ be locally Lipschitz at $x_{0}$. We define $\operatorname{Jac}(f, x)$ as the (nonempty) set of limit points of sequences $\left\{\nabla f\left(x_{k}\right)\right\}$ where $\left\{x_{k}\right\}$ is a sequence converging to $x_{0}$ and such that $f$ is Fréchet differentiable at $x_{k}$ with Jacobian $\nabla f\left(x_{k}\right)$. One can see ([9]), as a consequence of Rademacher's Theorem that $\operatorname{Jac}\left(f, x_{0}\right)$ is nonempty. Moreover the convex hull of $\operatorname{Jac}\left(f, x_{0}\right)$ is equal to the Clarke generalized Jacobian of $f$ at $x$.

Let $\mathrm{f}: \mathrm{U} \subseteq \mathbb{R}^{k} \rightarrow \mathbb{R}^{k}$ be a $\mathrm{PC}^{1}$ function (with selection functions $\mathrm{f}_{\mathrm{i}}$ ). The relation between the Bouligand derivative and the above generalized notion of Jacobian is clarified by the following formula [9, Lemma 2]:

$$
\operatorname{Jac}\left(f^{\prime}\left(x_{0}, \cdot\right), 0\right) \subseteq \operatorname{Jac}\left(f, x_{0}\right)=\left\{\nabla f_{i}\left(x_{0}\right): i \in \overline{\mathcal{I}}\left(x_{0}\right)\right\},
$$

where $\overline{\mathcal{I}}\left(x_{0}\right)=\left\{i: x_{0} \in \operatorname{cl} \operatorname{int}\{x \in U: i \in \mathcal{I}(x)\}\right\}$, see e.g. 6 .

The following two results of [9] play a crucial role in the following. Here, we slightly reformulate them to match our notation.

Theorem 2.3 (Thm. 4 of [9]). Let $\mathrm{f}: \mathrm{U} \subseteq \mathbb{R}^{k} \rightarrow \mathbb{R}^{k}$ be a $\mathrm{PC}^{1}$ function. Then $\mathrm{f}$ is a local Lipschitz homeomorphism at $\mathrm{x}_{0} \in \mathrm{U}$ if and only if $\mathrm{Jac}\left(\mathrm{f}, \mathrm{x}_{0}\right)$ consists of matrices whose determinants have the same nonzero sign and, for a sufficiently small neighbourhood $\mathrm{U}_{0}$ of $\mathrm{x}_{0}, \operatorname{deg}\left(\mathrm{f}, \mathrm{U}_{0}, 0\right)$ is well-defined and has value \pm 1 .

Theorem 2.4 (Thm. 5 of 9 ). Let $\mathrm{f}: \mathrm{U} \subseteq \mathbb{R}^{\mathrm{k}} \rightarrow \mathbb{R}^{\mathrm{k}}$ be a $\mathrm{PC}^{1}$ function, and let $\mathrm{x}_{0} \in \mathrm{U}$. Assume that

$$
\operatorname{Jac}\left(f, x_{0}\right)=J\left(f^{\prime}\left(x_{0}, \cdot\right), 0\right)
$$

then the following statements are equivalent:

(1) $\mathrm{f}$ is a local Lipschitz homeomorphism at $\mathrm{x}_{0} \in \mathrm{U}$;

(2) $f^{\prime}\left(x_{0}, \cdot\right)$ is bijective;

(3) $\mathrm{f}^{\prime}\left(\mathrm{x}_{0}, \cdot\right)$ is a (global) Lipschitz homeomorphism.

Moreover, if any of (i)-(iii) holds, then $\mathrm{f}$ is a local $\mathrm{PC}^{1}$ homeomorphism at $\mathrm{x}_{0}$.

We conclude this subsection recalling the classical notion of Bouligand tangent cone. Let $\mathrm{C} \subseteq \mathbb{R}^{\mathrm{k}}$ be a nonempty closed subset. Given $\chi \in \mathrm{C}$, the Bouligand 
tangent cone to $\mathrm{C}$ at $\mathrm{x}$ is the set:

$$
\left\{v \in \mathbb{R}^{k}: \exists \alpha_{j} \rightarrow 0^{+}, \exists v_{j} \rightarrow v \text { s.t. } x+\alpha_{j} v_{j} \in C\right\} .
$$

2.2. Topological degree. In this section we briefly recall the notion of Brouwer degree of a map and summarize some of its properties that will be used in the rest of the paper. Major references for this topic are, for instance, Milnor [8], Deimling [5] and Lloyd [7]; see also [3] for a quick introduction.

A triple $(f, U, p)$, with $p \in \mathbb{R}^{k}$ and $f$ a proper map defined in some neighbourhood of the open set $\mathrm{U} \subseteq \mathbb{R}^{k}$, is said to be admissible if $\mathrm{f}^{-1}(p) \cap \mathrm{U}$ is compact. Given an admissible triple $(f, u, p)$, it is defined an integer $\operatorname{deg}(f, u, p)$, called the degree of $f$ in $\mathrm{U}$ respect to $\mathrm{p}$, that in some sense counts (algebraically) the elements of $\mathrm{f}^{-1}(\mathrm{p})$ which lie in $U$. In fact, when in addition to the admissibility of $(f, U, p)$ we let $f$ be $C^{1}$ in a neighbourhood of $f^{-1}(p) \cap U$ and assume $p$ is a regular value of $f$, the set $f^{-1}(p) \cap \mathrm{U}$ is finite, and one has

$$
\operatorname{deg}(f, U, p)=\sum_{x \in f^{-1}(p) \cap u} \operatorname{sign} \operatorname{det}\left(f^{\prime}(x)\right),
$$

where $f^{\prime}(x)$ denotes the (Fréchet) derivative of $f$ at $x$. See e.g. 8 for a broader definition in the case when $(f, \mathcal{U}, p)$ is just an admissible triple.

The Brouwer degree enjoys many known properties only a few of which are needed in this paper. We now remind some of them.

(Excision.) If $(\mathrm{f}, \mathrm{U}, \mathrm{y})$ is admissible and $\mathrm{V}$ is an open subset of $\mathrm{U}$ such that $\mathrm{f}^{-1}(\mathrm{y}) \cap \mathrm{U} \subseteq \mathrm{V}$, then $(\mathrm{f}, \mathrm{V}, \mathrm{y})$ is admissible and

$$
\operatorname{deg}(f, u, y)=\operatorname{deg}(f, V, y) .
$$

(Boundary Dependence.) Let $\mathrm{U} \subseteq \mathbb{R}^{\mathrm{k}}$ be open, and let $\mathrm{f}$ and $\mathrm{g}$ be $\mathbb{R}^{\mathrm{k}}$-valued functions defined in a neighbourhood of $U$ be such that $f(x)=g(x)$ for all $x \in \partial U$. Assume that $\mathrm{U}$ is bounded or, more generally, that $f$ and $g$ are proper and the difference map $f-g: \overline{\mathrm{U}} \rightarrow \mathbb{R}^{k}$ has bounded image. Then

$$
\operatorname{deg}(\mathbf{f}, \mathbf{U}, \mathbf{y})=\operatorname{deg}(\mathbf{g}, \mathbf{u}, \mathbf{y})
$$

for any $y \in \mathbb{R}^{k} \backslash f(\partial u)$.

Observe that if $f: \mathbb{R}^{k} \rightarrow \mathbb{R}^{k}$ is proper then $\operatorname{deg}\left(f, \mathbb{R}^{k}, p\right)$ is well-defined for any $p \in \mathbb{R}^{k}$, moreover, by the above property, it is actually independent of the choice of $p$. In this case we shall simply write $\operatorname{deg}(f)$ instead of the more cumbersome $\operatorname{deg}\left(f, \mathbb{R}^{k}, p\right)$.

Finally, we mention a well-known integral formula for the computation of the degree of an admissible triple when the dimension of the space is $k=2$ (see e.g. [5, 7]) which we present here in a simplified form.

Assume that $f: \mathbb{R}^{2} \rightarrow \mathbb{R}^{2}$ is a proper map, let $B_{r} \subseteq \mathbb{R}^{2}$ be a ball of radius $r>0$ centered at the origin and let $S_{r}=r S^{1}=\partial B_{r}$. If $0 \notin f\left(S_{r}\right)$, then the degree of $f$ in $B_{r}$ relative to 0 coincides with the winding number of the curve $\sigma:[0,1] \rightarrow \mathbb{R}^{2}$ given by

$$
\sigma(t)=f(r \cos (2 \pi t), r \sin (2 \pi t)) .
$$

In other words,

$$
\operatorname{deg}\left(f, B_{r}, 0\right)=\frac{1}{2 \pi} \int_{f\left(S_{r}\right)} \omega
$$

where $\omega$ is the 1 -form

$$
\omega=\frac{x d y}{x^{2}+y^{2}}-\frac{y d x}{x^{2}+y^{2}}
$$


In fact, if $B_{r}$ is large enough to contain the compact set $f^{-1}(0)$, then

$$
\operatorname{deg}(f)=\frac{1}{2 \pi} \int_{f\left(S_{r}\right)} \omega
$$

\section{Piecewise Continuous linear maps and topological Degree}

Observe that any nondegenerate continuous strongly piecewise linear map $G$ is differentiable in $\mathbb{R}^{k} \backslash \cup_{i=1}^{n} \partial C_{i}$. It is easily shown that $G$ is proper, and therefore $\operatorname{deg}\left(G, \mathbb{R}^{k}, p\right)$ is well-defined for any $p \in \mathbb{R}^{k}$. In fact, one immediately checks that $\mathrm{G}^{-1}(0)=\{0\}$. So, as remarked above, we can write $\operatorname{deg}(\mathrm{G})$ in lieu of $\operatorname{deg}\left(\mathrm{G}, \mathbb{R}^{k}, p\right)$.

The following linear algebra result plays an important role in the paper.

Proposition 3.1. Let $\mathrm{A}$ and $\mathrm{B}$ be linear automorphisms of $\mathbb{R}^{\mathrm{k}}$. Assume that for some $v \in \mathbb{R}^{k} \backslash\{0\}, A$ and $B$ coincide on the space $\{v\}^{\perp}$. Then, the map $\mathcal{L}_{\mathrm{AB}}$ defined by $x \mapsto \mathrm{A} x$ if $\langle v, x\rangle \geq 0$, and by $\chi \mapsto \mathrm{B} x$ if $\langle v, x\rangle \leq 0$, is a homeomorphism if and only if $\operatorname{det}(A) \cdot \operatorname{det}(B)>0$.

Proof. Let $w_{1}, \ldots, w_{n-1}$ be a basis of the hyperplane $\{v\}^{\perp}$, then $w_{1}, \ldots, w_{n-1}, v$ is a basis of $\mathbb{R}^{n}$. The matrix of $A^{-1} B$ in this basis is given by

$$
\left(\begin{array}{c|c} 
& \gamma_{1} \\
\mathbf{I}_{n-1} & \vdots \\
& \gamma_{n-1} \\
\hline 0_{n-1}^{t} & \gamma_{n}
\end{array}\right)
$$

where $I_{n-1}$ is the $n-1$ unit matrix, $O_{n-1}$ is the $n-1$ null vector and the $\gamma_{i}$ 's are defined by

$$
A^{-1} B v=\sum_{i=1}^{n-1} \gamma_{i} w_{i}+\gamma_{n} v
$$

Thus $\gamma_{n}$ is positive if and only if $\operatorname{det}(A) \cdot \operatorname{det}(B)$ is positive.

Observe that if $\gamma_{n}$ is negative then $\mathcal{L}_{A B}$ is not one-to-one. In fact, being

$$
A w_{i}=B w_{i}, \forall i=1, \ldots, n-1, \text { and }\left\langle\sum_{i=1}^{n-1}-\frac{\gamma_{i}}{\gamma_{n}} w_{i}+\frac{1}{\gamma_{n}} v, v\right\rangle=\frac{\|v\|^{2}}{\gamma_{n}}<0,
$$

we get

$$
\begin{aligned}
\mathcal{L}_{\mathrm{AB}}(v)=A\left(\sum_{i=1}^{n-1}-\frac{\gamma_{i}}{\gamma_{n}} w_{i}+\frac{1}{\gamma_{n}} A^{-1} B v\right)=\sum_{i=1}^{n-1}-\frac{\gamma_{i}}{\gamma_{n}} A w_{i}+\frac{1}{\gamma_{n}} B v \\
=B\left(\sum_{i=1}^{n-1}-\frac{\gamma_{i}}{\gamma_{n}} w_{i}+\frac{1}{\gamma_{n}} v\right)=\mathcal{L}_{A B}\left(\sum_{i=1}^{n-1}-\frac{\gamma_{i}}{\gamma_{n}} w_{i}+\frac{1}{\gamma_{n}} v\right) .
\end{aligned}
$$

We now prove that $\mathcal{L}_{A B}$ is injective if $\gamma_{n}$ is positive. Assume this is not true. Since both $A$ and $B$ are invertible, there exist $z_{A}, z_{B} \in \mathbb{R}^{n}$ such that $\left\langle v, z_{A}\right\rangle>0$, $\left\langle v, z_{B}\right\rangle<0$ and $A z_{A}=B z_{B}$ or, equivalently, $A^{-1} B z_{B}=z_{A}$. Let

$$
z_{A}=\sum_{i=1}^{n-1} c_{A}^{i} w_{i}+c_{A} v, \quad z_{B}=\sum_{i=1}^{n-1} c_{B}^{i} w_{i}+c_{B} v .
$$

Clearly $c_{A}>0, c_{B}<0$. The equality $A^{-1} B z_{B}=z_{A}$ is equivalent to

$$
\sum_{i=1}^{n-1} c_{B}^{i} w_{i}+c_{B} \sum_{i=1}^{n-1} \gamma_{i} w_{i}+c_{B} \gamma_{n} v=\sum_{i=1}^{n-1} c_{A}^{i} w_{i}+c_{A} v
$$


Consider the scalar product with $v$, we get $c_{B} \gamma_{n}\|v\|^{2}=c_{A}\|v\|^{2}$, which is a contradiction.

We finally prove that, if $\gamma_{n}$ is positive, then $\mathcal{L}_{A B}$ is surjective. Let $z \in \mathbb{R}^{n}$. There exist $y_{A}, y_{B} \in \mathbb{R}^{n}$ such that $A y_{A}=B y_{B}=z$. If either $\left\langle v, y_{A}\right\rangle \geq 0$ or $\left\langle v, \mathrm{y}_{B}\right\rangle \leq 0$, there is nothing to prove. Let us assume $\left\langle v, \mathrm{y}_{A}\right\rangle<0$ and $\left\langle v, \mathrm{y}_{B}\right\rangle>0$. In this case $A^{-1} B y_{B}=y_{A}$ and proceeding as above we get a contradiction.

Corollary 3.2. Let A, B and $v$ be as in Proposition 3.1. Define $\mathcal{L}_{\mathrm{AB}}$, as in Proposition 3.1, by

$$
\mathcal{L}_{\mathrm{AB}}(x)= \begin{cases}\mathrm{A} x & \text { if }\langle v, x\rangle \geq 0 \\ \mathrm{~B} x & \text { if }\langle v, x\rangle \leq 0\end{cases}
$$

Assume that $\operatorname{det}(A) \cdot \operatorname{det}(B)>0$. Then $\operatorname{deg}\left(\mathcal{L}_{A B}\right)=\operatorname{sign} \operatorname{det}(A)=\operatorname{sign} \operatorname{det}(B)$.

Proof. The map $\mathcal{L}_{\mathrm{AB}}$ is invertible by Proposition 3.1. Take any $p \in \mathbb{R}^{k}$ such that the singleton $\{\mathbf{q}\}=\mathcal{L}_{\mathrm{AB}}^{-1}(\mathrm{p})$ does not belong to $v^{\perp}$. Then, Formula 2.3 yields the assertion.

Another useful tool for the computation of the topological degree of a strongly piecewise linear map is the following lemma:

Lemma 3.3. If $\mathrm{G}$ is a continuous strongly piecewise linear map as in Definition 2.2 with $\operatorname{det}\left(\mathrm{L}_{\mathrm{i}}\right)>0, \forall \mathrm{i}=1, \ldots, \mathrm{n}$, then $\operatorname{deg}(\mathrm{G})>0$. In particular, if there exists $\mathrm{q} \neq 0$ whose preimage $\mathrm{G}^{-1}(\mathrm{q})$ is a singleton that belongs to at most two of the convex polyhedral cones $\mathrm{C}_{\mathfrak{i}}$, then $\operatorname{deg}(\mathrm{G})=1$.

Proof. Let us assume in addition that $\mathrm{q} \notin \cup_{i=1}^{n} \mathrm{G}\left(\partial \mathrm{C}_{i}\right)$. Observe that the set $\cup_{i=1}^{n} G\left(\partial C_{i}\right)$ is nowhere dense hence $A:=G\left(C_{1}\right) \backslash \cup_{i=1}^{n} G\left(\partial C_{i}\right)$ is non-empty. Take $x \in A$ and observe that if $y \in G^{-1}(x)$ then $y \notin \cup_{i=1}^{n} \partial C_{i}$. Thus, by (2.3),

$$
\operatorname{deg}(\mathrm{G})=\sum_{y \in \mathrm{G}^{-1}(x)} \operatorname{sign} \operatorname{det} \mathrm{G}^{\prime}(\mathrm{y})=\# \mathrm{G}^{-1}(x)
$$

Since $\mathrm{G}^{-1}(x) \neq \emptyset \operatorname{deg}(\mathrm{G})>0$.

We now consider the second part of the assertion. Assume in addition that $q \notin \cup_{i=1}^{n} G\left(\partial C_{i}\right)$. Taking $x=q$ in 3.1 we $\operatorname{get} \operatorname{deg}(G)=1$.

Let us now remove the additional assumption. Let $\{p\}=\mathrm{G}^{-1}(\mathrm{q})$ be such that $p \in \partial C_{i} \cap \partial C_{j}$ for some $i \neq j$. Observe that by assumption $p \neq 0$ does not belong to any cone $\partial C_{s}$ for $s \notin\{i, j\}$. Thus one can find a neighborhood $V$ of $p$, with $V \subset \operatorname{int}\left(C_{i} \cup C_{j} \backslash\{0\}\right)$. By the excision property of the topological degree $\operatorname{deg}(G)=\operatorname{deg}(G, V, p)$. Let $\mathcal{L}_{L_{i} L_{j}}$ be a map as in Proposition 3.1. Observe that, by Corollary 3.2. the assumption on the signs of the determinants of $L_{i}$ and $L_{j}$ imply that $\operatorname{deg}\left(\mathcal{L}_{\mathrm{L}_{i} \mathrm{~L}_{j}}\right)=1$. Also notice that $\left.\mathcal{L}_{\mathrm{L}_{i} \mathrm{~L}_{j}}\right|_{\partial \mathrm{V}}=\left.\mathrm{G}\right|_{\partial \mathrm{V}}$. Hence, by the excision and boundary dependence properties of the degree we have

$$
1=\operatorname{deg}\left(\mathcal{L}_{\mathrm{L}_{i} \mathrm{~L}_{j}}\right)=\operatorname{deg}\left(\mathcal{L}_{\mathrm{L}_{\mathrm{i}} \mathrm{L}_{j}}, \mathrm{~V}, \mathrm{p}\right)=\operatorname{deg}(\mathrm{G}, \mathrm{V}, \mathrm{p}) .
$$

Thus, $\operatorname{deg}(\mathrm{G})=1$ as claimed.

Remark 3.4. One can show that if $\operatorname{det}\left(\mathrm{L}_{\mathrm{i}}\right)<0$, for all $\mathrm{i}=1, \ldots, \mathrm{n}$, then

$$
\operatorname{deg}(\mathrm{G})<0 \text {. }
$$

In particular, if there exists $\mathbf{q} \neq 0$ whose preimage $\mathrm{G}^{-1}(\mathbf{q})$ is a singleton that belongs to at most two of the convex cones $\mathrm{C}_{i}$, then $\operatorname{deg}(\mathrm{G})=-1$. To see this, it is enough to compose $\mathrm{G}$ with the permutation matrix

$$
\mathrm{P}=\left(\begin{array}{cc}
\mathrm{J} & 0 \\
0 & \mathbf{I}_{\mathrm{n}-2}
\end{array}\right), \quad \mathrm{J}:=\left(\begin{array}{ll}
0 & 1 \\
1 & 0
\end{array}\right),
$$

and $\mathbf{I}_{\mathrm{n}-2}$ is the $(\mathrm{n}-2) \times(\mathrm{n}-2)$ identity matrix. 
We conclude this section by observing that if $\mathrm{G}$ is a nondegenerate continuous strongly piecewise linear map in $\mathbb{R}^{2}$ then, by (2.4),

$$
\operatorname{deg}(\mathrm{G})=\frac{1}{2 \pi} \int_{\mathrm{G}_{\left(\mathrm{S}^{1}\right)}} \omega .
$$

(observe, in fact, that $\mathrm{G}^{-1}(0)=\{0\}$ ). This formula plays an important role in what follows.

\section{Main Results: inVERTIBILITy OF PIECEWISE LiNEAR MAPS}

We now turn to our main scope that is invertibility of continuous strongly piecewise linear maps. We begin with a relatively simple result.

Proposition 4.1. Let $\mathrm{G}$ be continuous strongly piecewise linear. If $\mathrm{G}$ is invertible, then it is nondegenerate.

Proof. Let $C_{i}, i=1, \ldots, n$ be the polyhedral cones decomposition of $\mathbb{R}^{n}$ relative to $G$ and let $L_{i}=\left.G\right|_{C_{i}}$. We need to show that $\operatorname{det}\left(L_{i}\right) \neq 0$ for any $i=1, \ldots, n$ and that all these determinants have the same sign.

We first prove that no such determinant is null. Assume by contradiction that, for some $i \in\{1, \ldots, n\}$, $\operatorname{det}\left(L_{i}\right)=0$. Without loss of generality we may assume $i=1$. Let $v \in \operatorname{ker}\left(\mathrm{L}_{1}\right) \backslash\{0\}$. If $v \in \mathrm{C}_{1}$, then $\mathrm{G}(v)=\mathrm{L}_{1} v=0=\mathrm{G}(0)$, so that $\mathrm{G}$ is not injective. A contradiction. If $v \notin C_{1}$, then there exist $w \in \operatorname{int}\left(C_{1}\right)$ and $\lambda \in \mathbb{R}$, $\lambda \neq 0$ such that $w+\lambda v \in \operatorname{int}\left(\mathrm{C}_{1}\right)$. Thus $\mathrm{G}(w+\lambda v)=\mathrm{L}_{1}(w+\lambda v)=\mathrm{L}_{1} w=\mathrm{G}(w)$, so that $G$ is, also in this case, not injective. These contradictions show that the determinants $\operatorname{det}\left(\mathrm{L}_{\mathrm{i}}\right)$ 's cannot be zero.

We now show that all these determinants have the same sign. As in the first part of the proof, we proceed by contradiction. Let

$$
S:=\left\{C_{i} \cap C_{j}: i, j \in\{1, \ldots, n\}, \operatorname{codim} \operatorname{span}\left(C_{i} \cap C_{j}\right) \geq 2\right\} .
$$

Notice that when the dimension $k$ of the ambient space $\mathbb{R}^{k}$ is 1 , then $S=\emptyset$, and if $k=2$ then $S$ is merely the origin. Assume by contradiction that there are $i, j \in\{1, \ldots, n\}$ such that $\operatorname{det}\left(L_{i}\right) \operatorname{det}\left(L_{j}\right)<0$. Since $\mathbb{R}^{k} \backslash S$ is arcwise connected, it is not difficult to prove that, there must exist two cones $C_{\bar{i}}$ and $C_{\bar{j}}$ such that codim $\operatorname{span}\left(\mathrm{C}_{\bar{i}} \cap \mathrm{C}_{\bar{j}}\right)=1$ and $\operatorname{det}\left(\mathrm{L}_{\overline{\mathrm{i}}}\right) \operatorname{det}\left(\mathrm{L}_{\bar{j}}\right)<0$. Without any loss of generality we may assume $\bar{i}=1, \bar{j}=2$. Let $v \in \mathbb{R}^{k}$ such that $\operatorname{span}\left(C_{1} \cap C_{2}\right)=v^{\perp}, C_{1} \subset$ $\left\{x \in \mathbb{R}^{k}:\langle v, x\rangle \geq 0\right\}, C_{2} \subset\left\{x \in \mathbb{R}^{k}:\langle v, x\rangle \leq 0\right\}$. Let $w_{1}, w_{2}, \ldots, w_{n-1}$ be a basis for $\operatorname{span}\left(\mathrm{C}_{1} \cap \mathrm{C}_{2}\right)$ such that

$$
\left\{\sum_{i=1}^{n-1} c_{i} w_{i}: c_{i} \geq 0 \quad i=1, \ldots, n-1\right\} \subseteq\left(C_{1} \cap C_{2}\right),
$$

and let

$$
\mathrm{L}_{1}^{-1} \mathrm{~L}_{2} v=\gamma_{\mathrm{n}} v+\sum_{i=1}^{\mathrm{n}-1} \gamma_{\mathrm{i}} w_{\mathrm{i}}
$$

As in the proof of Proposition 3.1 one can show that $\gamma_{n}<0$. Take $c_{1}, \ldots, c_{n-1}>0$ and define

$$
z_{1}:=v+\sum_{i=1}^{n-1} c_{i} w_{i} \quad \text { and } \quad z_{2}:=\frac{1}{\gamma_{n}} v+\sum_{i=1}^{n-1}\left(c_{i}-\frac{\gamma_{i}}{\gamma_{n}}\right) w_{i} .
$$

An easy computation shows that $\mathrm{L}_{1} z_{1}=\mathrm{L}_{2} z_{2}$. Choosing $\mathrm{c}_{1}, \ldots, \mathrm{c}_{\mathrm{n}-1}$ large enough, we can assume that $z_{1} \in \mathrm{C}_{1}, z_{2} \in \mathrm{C}_{2}$. Thus $\mathrm{G}\left(z_{1}\right)=\mathrm{G}\left(z_{2}\right)$, i.e. $\mathrm{G}$ is not injective, against the assumption. This contradiction shows that all $\operatorname{determinants} \operatorname{det}\left(\mathrm{L}_{s}\right)$, $s \in\{1, \ldots, n\}$, share the same sign. 
Simple considerations (e.g. Examples 4.6 and 4.7below) show that the converse of Propositions 4.1] is not true in general. In order to partially invert this proposition, different situations must be considered. We begin with a simple consequence of Lemma 3.3 .

Theorem 4.2. Let $\mathrm{G}: \mathbb{R}^{k} \rightarrow \mathbb{R}^{k}$ be a continuous strongly piecewise linear map as in Definition 2.2 with $\operatorname{det}\left(\mathrm{L}_{\mathrm{i}}\right)$ of constant sign for all $\mathrm{i}=1, \ldots, \mathrm{n}$. Assume also that there exists $\mathrm{q} \in \mathbb{R}^{k}$ whose preimage $\mathrm{G}^{-1}(\mathrm{q})$ is a singleton that belongs to at most two of the polyhedral cones $\mathrm{C}_{i}$. Then $\mathrm{G}$ is a Lipschitz homeomorphism.

Proof. Lemma 3.3 and Remark 3.4 imply that $\operatorname{deg}(\mathrm{G})= \pm 1$. The assertion follows from Theorem 2.3 and Lemma 2.1

Remark 4.3. The condition in Theorem 4.2 concerning the existence of a point $\mathrm{q}$ whose preimage is a singleton belonging to at most two polyhedral cones, is equivalent to the existence of a half-line at the origin whose preimage is a single half-line. In fact, as a consequence of Theorem 4.2, one has that if the determinants $\operatorname{det}\left(\mathrm{L}_{\mathrm{i}}\right)$ have constant sign for all $\mathrm{i}=1, \ldots, \mathrm{n}$ the existence of such a half-line implies that all the half-lines at the origin must have the same property.

Remark 4.4. Observe that the only nontrivial (i.e. such that are not reducible to linear maps) continuous strongly piecewise linear maps with $\mathrm{n}=2$ are those in which the cones are half-spaces. In fact, two linear endomorphisms of $\mathbb{R}^{k}$ that agree on two linearly independent vectors, necessarily coincide. Hence, when $\mathfrak{n}=2$, it is sufficient to consider the case when the two nontrivial cones are half-spaces. This has already been done in Proposition 3.1.

The point $\mathrm{q}$ in Theorem 4.2 may be difficult to determine if the linear maps $\mathrm{L}_{i}$ 's are given in a complicate way. However, in some cases, invertibility of continuous nondegenerate strongly piecewise linear maps can be deduced merely from their nondifferentiability structure. The easiest nontrivial case, i.e. when $n=2$, has already been treated (Proposition 13.1) in arbitrary dimension just by means of linear algebra. The other cases, $n=3$ and $n=4$, will be investigated in dimension $\mathrm{k}=2$ only.

We are now in a position to state our main result concerning the invertibility of continuous strongly piecewise linear maps in $\mathbb{R}^{2}$.

Theorem 4.5. Let $\mathrm{G}: \mathbb{R}^{2} \rightarrow \mathbb{R}^{2}$ be as in Definition 2.2. We have that, if one of the following conditions holds:

(1) $n \in\{1,2,3\}$;

(2) $\mathrm{n}=4$ and all the cones are convex;

then $\mathrm{G}$ has a continuous piecewise linear inverse.

Before we provide the proof of this result, we show with two examples that the assumptions of Theorem 4.5 are, to some extent, sharp.

Our first example shows that for $n>4$ there are G's as above that are not invertible even if the cones are convex.

Example 4.6. Consider a nondegenerate continuous piecewise linear map $\mathrm{G}: \mathbb{R}^{2} \rightarrow$ $\mathbb{R}^{2}$ defined as in Definition 2.2 by

$$
\begin{aligned}
& \mathrm{L}_{1}=\left(\begin{array}{cc}
1 & -\sqrt{2} \\
0 & \sqrt{2}-1
\end{array}\right) \quad \mathrm{L}_{2}=\left(\begin{array}{cc}
-\sqrt{2} & -\sqrt{2}+1 \\
1 & 0
\end{array}\right) \\
& \mathrm{L}_{3}=\left(\begin{array}{cc}
0 & 1 \\
-\sqrt{2}+1 & -\sqrt{2}
\end{array}\right) \quad \mathrm{L}_{4}=\left(\begin{array}{cc}
\sqrt{2}-1 & 0 \\
-\sqrt{2} & 1
\end{array}\right) \quad \mathrm{L}_{5}=\left(\begin{array}{ll}
1 & 0 \\
0 & 1
\end{array}\right)
\end{aligned}
$$




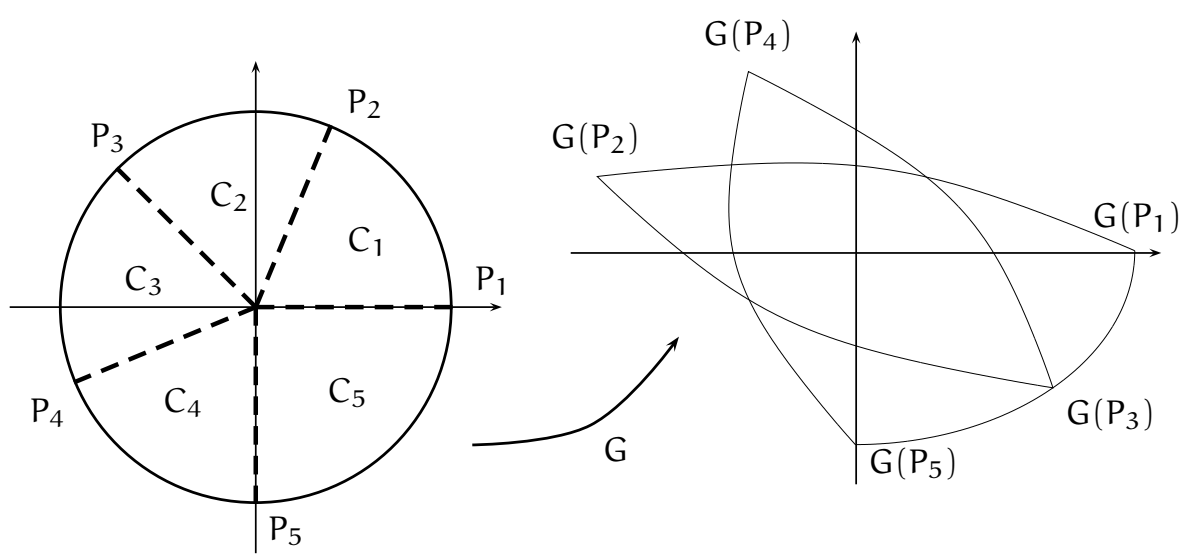

Figure 1. The image of $S^{1}$ under $\mathrm{G}$ in Example 4.6. For clarity's sake, the radial distance of $\mathrm{G}\left(\mathrm{S}^{1}\right)$ from $(0,0)$ has been rescaled.

where the corresponding cones are given, in polar coordinates, by the pairs $(\rho, \theta)$ with arbitrary $\rho$ 's and $\theta$ chosen as in the following table:

\begin{tabular}{|c|c|c|c|c|}
\hline $\mathrm{C}_{1}$ & $\mathrm{C}_{2}$ & $\mathrm{C}_{3}$ & $\mathrm{C}_{4}$ & $\mathrm{C}_{5}$ \\
\hline $0 \leq \theta \leq \frac{3}{8} \pi$ & $\frac{3}{8} \pi \leq \theta \leq \frac{3}{4} \pi$ & $\frac{3}{4} \pi \leq \theta \leq \frac{9}{8} \pi$ & $\frac{9}{8} \pi \leq \theta \leq \frac{3}{2} \pi$ & $\frac{3}{2} \pi \leq \theta \leq 2 \pi$ \\
\hline
\end{tabular}

This map is illustrated in Figure 1. As the picture suggests, the above defined map $\mathrm{G}$ is not invertible because it is not injective.

Our second example shows an instance of noninvertible $G$ with $n=4$ and one nonconvex cone.

Example 4.7. Consider $\mathrm{G}: \mathbb{R}^{2} \rightarrow \mathbb{R}^{2}$ as in Definition [2.2, with

$$
\mathrm{L}_{1}=\left(\begin{array}{ll}
1 & 0 \\
0 & 1
\end{array}\right), \quad \mathrm{L}_{2}=\left(\begin{array}{cc}
1 & 0 \\
2 \sqrt{3} & 1
\end{array}\right), \quad \mathrm{L}_{3}=\left(\begin{array}{cc}
-2 & -\sqrt{3} \\
-\sqrt{3} & -2
\end{array}\right), \quad \mathrm{L}_{4}=\left(\begin{array}{cc}
1 & 2 \sqrt{3} \\
0 & 1
\end{array}\right),
$$

and the cones are given, in polar coordinates, by the pairs $(\rho, \theta)$ with arbitrary $\rho$ 's and $\theta$ chosen as in the following table:

\begin{tabular}{|c|c|c|c|}
\hline$C_{1}$ & $C_{2}$ & $C_{3}$ & $C_{4}$ \\
\hline $0 \leq \theta \leq \frac{\pi}{2}$ & $\frac{\pi}{2} \leq \theta \leq \frac{2}{3} \pi$ & $\frac{2}{3} \pi \leq \theta \leq \frac{11}{6} \pi$ & $\frac{11}{6} \pi \leq \theta \leq 2 \pi$ \\
\hline
\end{tabular}

Figure 2 illustrates this map. As the picture suggests, $\mathrm{G}$ defined as above is not injective and therefore it is not invertible.

Let us now turn to the task of proving Theorem 4.5. The proof is done differently according to the number of nontrivial cones in which the plane is pie-sliced. The proof, in the cases of $n=2$, boils down to Proposition 3.1 whereas the cases $n=3$ and $n=4$ will be treated with the help of Theorem 2.4. In order to apply this theorem it is necessary to estimate the topological degree of our map G. This will be done by the means of geometric considerations. The proof of the following lemma is based on an elementary linear algebra argument and is left to the reader.

Lemma 4.8. Let $\mathrm{A}: \mathbb{R}^{2} \rightarrow \mathbb{R}^{2}$ be linear and nonsingular and let $\mathrm{C} \in \mathbb{R}^{2}$ be a cone with vertex at the origin. Then $\mathrm{A}(\mathrm{C}) \subseteq \mathbb{R}^{2}$ is a cone with with vertex at the origin and the following statements hold:

(1) If C does not contain a half-plane, then $\mathrm{A}(\mathrm{C})$ is strictly convex. 


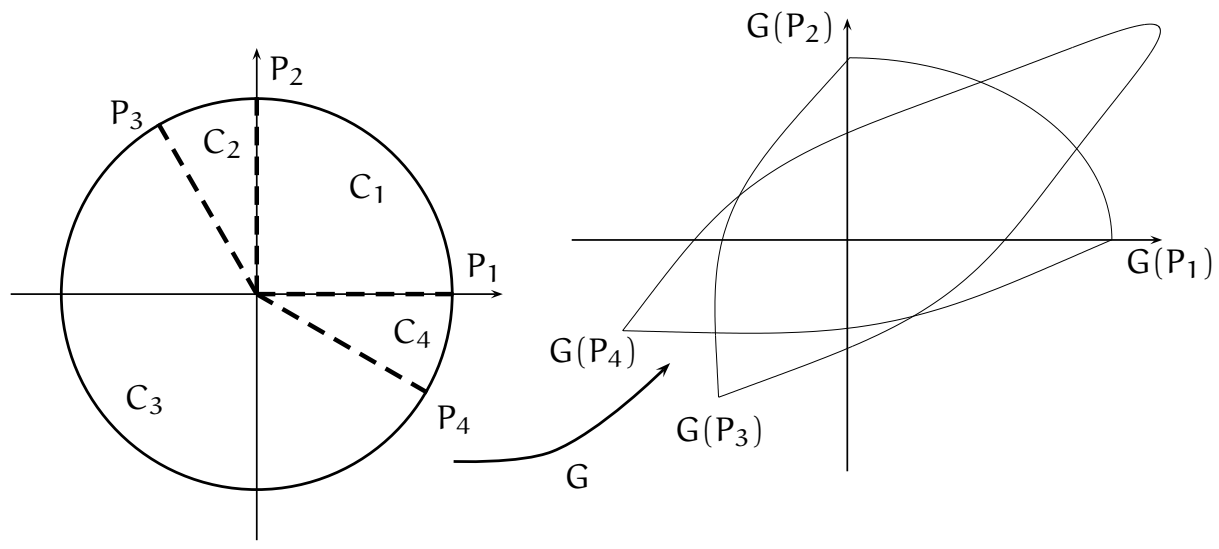

Figure 2. The image of $S^{1}$ under $G$ in Example 4.7. For clarity's sake, the radial distance of $\mathrm{G}\left(\mathrm{S}^{1}\right)$ from $(0,0)$ has been rescaled.

(2) If $\mathrm{C} \varsubsetneqq \mathbb{R}^{2}$ contains a half-plane, then so does $\mathrm{A}(\mathrm{C}) \varsubsetneqq \mathbb{R}^{2}$.

This lemma has an useful consequence:

Lemma 4.9. Let $\mathrm{A}: \mathbb{R}^{2} \rightarrow \mathbb{R}^{2}$ be linear and nonsingular and let $\mathrm{C} \varsubsetneqq \mathbb{R}^{2}$ be a cone with vertex at the origin. Let $\Gamma$ be the image of the arc $S^{1} \cap \mathrm{C}$. Then,

$$
\left|\int_{\Gamma} \omega\right| \in \begin{cases}{[0, \pi)} & \text { if } \mathrm{C} \text { does not contain a half-plane, } \\ {[\pi, 2 \pi)} & \text { otherwise. }\end{cases}
$$

In particular, we have that $\left|\int_{\Gamma} \omega\right|<2 \pi$ and $\left|\int_{\Gamma} \omega\right|<\pi$ when $C$ is strictly convex.

Proof. Observe first that by Lemma 4.8 there exists a half-line $s$ starting at the origin that does not intersect $A(C)$. Clearly the differential form $\omega$ is exact in $\mathbb{R}^{2} \backslash s$. Let $P_{1}$ and $P_{2}$ be the intersections of $\partial C$ with $S^{1}$. The path integral that appears in (4.1) does not depend on the chosen path connecting $A\left(p_{1}\right)$ and $A\left(p_{2}\right)$. With the choice of an appropriate path, for instance, the concatenation of a circular arc of radius $\left|A\left(P_{2}\right)\right|$ from $A\left(P_{2}\right)$ with the radial segment through $A\left(P_{1}\right)$ (see Figure (3)), it is not difficult to show that $\left|\int_{\Gamma} \omega\right|$ is merely the angular distance (we consider the angle that does not contain the half-line $s)$ between $A\left(p_{1}\right)$ and $A\left(p_{2}\right)$ as seen from the origin. The assertion now follows from Lemma 4.8 .

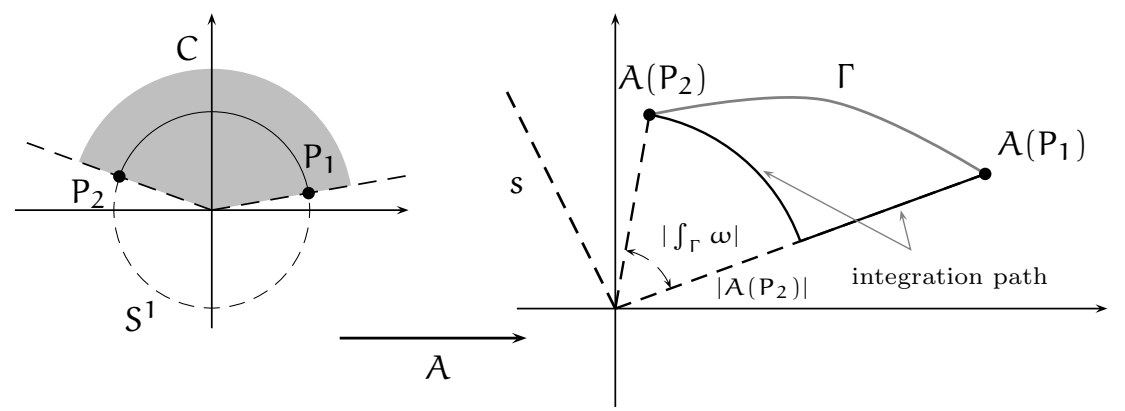

FigURE 3. The integration path in Lemma 4.9 
Lemma 4.10. Let $\mathrm{G}$ be as in Theorem 4.5 with $\mathrm{n}=3$ and $\operatorname{det} \mathrm{L}_{\mathrm{i}}>0, \forall i=1,2,3$. Then, $\operatorname{deg}(\mathrm{G})=1$.

Proof. We consider the two possible cases: when all the cones are strictly convex and when there is one cone containing a half-plane. For $i=1,2,3$, let $\Gamma_{i}$, be the image $G\left(S^{1} \cap C_{i}\right)$. In the first case, by Lemma 4.9 we have that $\int_{\Gamma_{i}} \omega<\pi$ for $i=1,2,3$. Hence, by Lemma 3.3 and formula (3.2),

$$
0<\operatorname{deg}(\mathrm{G})<\frac{\pi+\pi+\pi}{2 \pi}=\frac{3}{2} .
$$

Which, the degree being an integer, implies $\operatorname{deg}(\mathrm{G})=1$.

In the second case, only one of the cones, say $C_{1}$, may contain a half-plane. Thus, by Lemma 4.9, we have that $\int_{\Gamma_{1}} \omega<2 \pi$ and $\int_{\Gamma_{i}} \omega<\pi$ for $i=2,3$. Hence, inequality (4.2) becomes

$$
0<\operatorname{deg}(\mathrm{G})<\frac{2 \pi+\pi+\pi}{2 \pi}=2 .
$$

Which, again, implies $\operatorname{deg}(\mathrm{G})=1$.

Lemma 4.11. Let $\mathrm{G}$ be as in Theorem 4.5 with $\mathrm{n}=4$ and $\operatorname{det} \mathrm{L}_{\mathrm{i}}>0 \forall \mathrm{i}=1,2,3,4$. Then $\operatorname{deg}(\mathrm{G})=1$.

Proof. For $i=1, \ldots, 4$, let $\Gamma_{i}$, be the image $\mathrm{G}\left(\mathrm{S}^{1} \cap \mathrm{C}_{i}\right)$. By Lemma 4.9 we have that $\int_{\Gamma_{i}} \omega<\pi$ for $i=1, \ldots, 4$ since all the cones are convex. Hence, by Lemma 3.3 and formula (3.2),

$$
0<\operatorname{deg}(\mathrm{G})<\frac{\pi+\pi+\pi+\pi}{2 \pi}=2 .
$$

Which, the degree being an integer, $\operatorname{implies} \operatorname{deg}(\mathrm{G})=1$.

Remark 4.12. If $\operatorname{det} \mathrm{L}_{\mathrm{i}}<0 \forall \mathrm{i}=1, \ldots, \mathrm{n}$, composing $\mathrm{G}$ with the linear maps whose matrix in the standard basis of $\mathbb{R}^{2}$ is $\mathrm{J}=\left(\begin{array}{ll}0 & 1 \\ 1 & 0\end{array}\right)$, we get that $\mathrm{JG}: \mathbb{R}^{2} \rightarrow \mathbb{R}^{2}$ is one-to-one so that $\mathrm{G}$ is invertible as well.

We are now in a position to prove our main theorem.

Proof of Theorem 4.5. (Case $\mathrm{n}=1$.) In this case $\mathrm{G}$ is linear with nonzero determinant. Thus, there is nothing to prove.

(Case $n=2$.) See Proposition 3.1 and Remark 4.4.

(Cases $n=3$ and $n=4$.) In both cases, it follows from Lemmas 4.10 and 4.11 that $\operatorname{deg}(\mathrm{G})=1$, then the assertion follows from Theorem 2.3 .

Example 4.13. Let us consider a decomposition of the space $\mathbb{R}^{3}$ into four convex wedges $\mathrm{C}_{1}, \ldots, \mathrm{C}_{4}$ with a common edge along the straight line $\mathrm{r}$. Let $\mathrm{G}: \mathbb{R}^{3} \rightarrow \mathbb{R}^{3}$ be a continuous strongly piecewise linear map with respect to this decomposition and with

$$
\mathrm{G}(x)=\mathrm{L}_{i} x, \quad x \in \mathrm{C}_{i}, i=1, \ldots, 4
$$

and assume that det $\mathrm{L}_{i}$ share the same sign for $\mathrm{i}=1, \ldots, 4$. Then, as a consequence of Theorem 4.5. we have that $\mathrm{G}$ is invertible with continuous strongly piecewise linear inverse. To see that, consider a plane $\mathrm{P}$ orthogonal to $\mathrm{r}$. Clearly, the restriction $\left.\mathrm{G}\right|_{\mathrm{P}}$ is invertible by Theorem 4.5. Similarly, since $\mathrm{G}\left(\mathrm{x}_{\mathrm{r}}\right)=\mathrm{L}_{1} \mathrm{x}_{\mathrm{r}}=\ldots=\mathrm{L}_{4} \mathrm{x}_{\mathrm{r}}$, for any point $\mathrm{x}_{\mathrm{r}} \in \mathrm{r}$, and the $\mathrm{L}_{\mathrm{i}}$ 's are isomorphisms, $\mathrm{G}$ is invertible on $\mathrm{r}$. Given any vector $\mathrm{y} \in \mathbb{R}^{3}$, we can obtain $\mathrm{G}^{-1}(\mathrm{y})$ by the following argument. Write $\mathrm{y}=\mathrm{y}_{\mathrm{P}}+\mathrm{y}_{\mathrm{r}}$ where $\mathrm{y}_{\mathrm{P}}$ and $\mathrm{y}_{\mathrm{r}}$ denote the orthogonal projections of $\mathrm{y}$ onto $\mathrm{P}$ and $\mathrm{r}$, respectively. Then one has

$$
\mathrm{G}^{-1}(\mathrm{y})=\mathrm{L}_{1}^{-1}(\mathrm{y})+\left(\left.\mathrm{G}\right|_{\mathrm{P}}\right)^{-1}(\mathrm{y}) \text {. }
$$


We conclude this section with an example showing that our main result (at least when $n=4$ ) cannot be deduced from the well-known Clarke's Theorem. In fact, we exhibit a continuous piecewise linear map which is invertible by our main result although it does not satisfy the assumptions of Clarke's Theorem.

Example 4.14. Consider a continuous piecewise linear map $\mathrm{G}: \mathbb{R}^{2} \rightarrow \mathbb{R}^{2}$ defined as in Definition 2.2 by

$$
\mathrm{L}_{1}=\left(\begin{array}{ll}
1 & 0 \\
0 & 1
\end{array}\right), \quad \mathrm{L}_{2}=\left(\begin{array}{cc}
\frac{1}{10} & 0 \\
-10 & 1
\end{array}\right), \quad \mathrm{L}_{3}=\left(\begin{array}{rr}
\frac{5}{100} & \frac{5}{100} \\
-\frac{455}{100} & -\frac{445}{100}
\end{array}\right), \quad \mathrm{L}_{4}=\left(\begin{array}{cc}
1 & 1 \\
0 & \frac{1}{10}
\end{array}\right)
$$

where the corresponding cones are the quadrants $I, \ldots, I V$, respectively. This map is illustrated in Figure 4. As the picture suggests, G has degree 1. (The map G has been found with the help of a short FORTRAN program that assisted us in sifting many potential examples.)

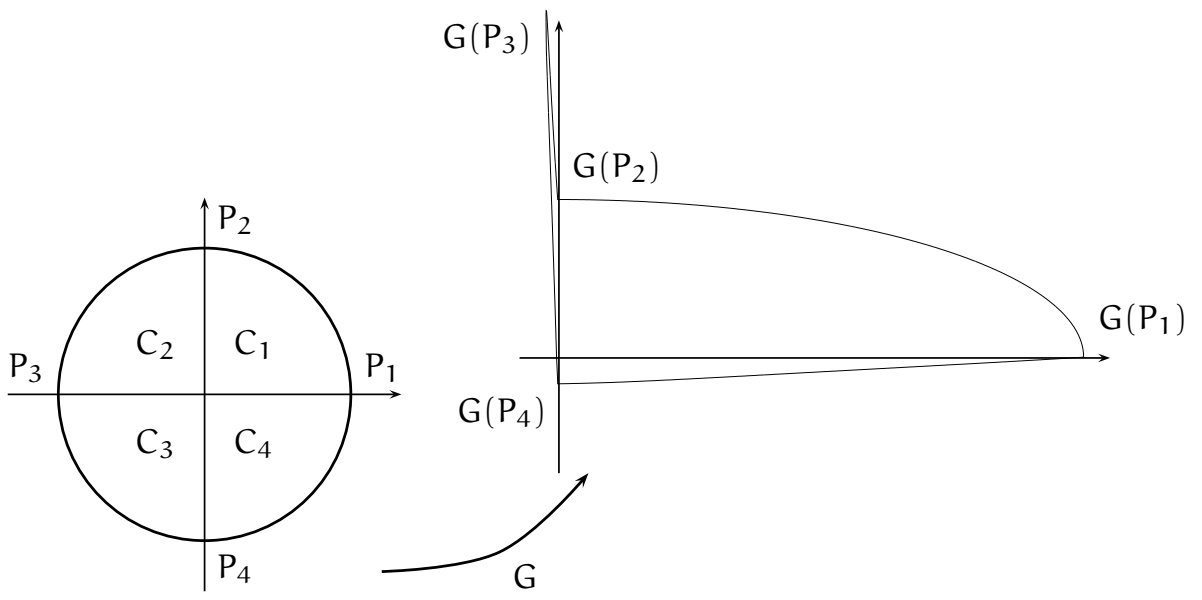

Figure 4. The image of $S^{1}$ under $\mathrm{G}$ in Example 4.14. For clarity's sake, the radial distance of $\mathrm{G}\left(\mathrm{S}^{1}\right)$ from $(0,0)$ and the proportion between axes have been both altered.

\section{Applichtion: Piecewise differentiable functions}

We now provide some applications of the results of the previous section to the local invertibility of $\mathrm{PC}^{1}$ functions. The basis for our considerations is the following consequence of Theorem 2.3 .

Theorem 5.1. Let $\mathrm{f}$ be an $\mathbb{R}^{\mathrm{k}}$-valued $\mathrm{PC}^{1}$ function in a neighborhood of $\mathrm{x}_{0} \in \mathbb{R}^{\mathrm{k}}$. Assume that

(1) All the elements of the geralized Jacobian of $f$ at $x_{0}$ have the same sign;

(2) The Bouligand differential of $\mathrm{f}$ at $\mathrm{x}_{0}$ is an invertible piecewise linear map.

Then $\mathrm{f}$ is locally invertible at $\mathrm{x}_{0}$.

Proof. It is not difficult to show that since $f^{\prime}\left(x_{0}, \cdot\right)$ is invertible,

$$
\operatorname{deg}\left(f^{\prime}\left(x_{0}, \cdot\right), V, 0\right)=\mathfrak{s},
$$

where $\mathfrak{s}$ denotes the common sign of the elements of the generalized Jacobian of $f$ at $x_{0}$

We claim that in a sufficiently small neighborhood $V$ of $x_{0}$ the map $f$ is admissibly homotopic to $f^{\prime}\left(x_{0}, \cdot\right)$ so that, by homotopy invariance, $\operatorname{deg}(f, V, 0)=\mathfrak{s}$. The assertion follows from Theorem 2.3 . 
We now prove the claim. Consider the homotopy

$$
H(x, \lambda)=f\left(x-x_{0}\right)+\lambda\left|x-x_{0}\right| \varepsilon\left(x-x_{0}\right), \quad \lambda \in[0,1],
$$

and let $\{\boldsymbol{i}=1, \ldots, k\}$ be the active index set of $f$ at $x_{0}$. Observe that

$$
m:=\inf \{|f(v)|:|v|=1\}=\min _{i=1, \ldots, k}\left\|d f_{i}\right\|>0 \text {. }
$$

Thus,

$$
|H(x, \lambda)| \geq\left(m-\left|\varepsilon\left(x-x_{0}\right)\right|\right)\left|x-x_{0}\right| .
$$

This shows that in a conveniently small ball centered at $x_{0}$, homotopy $H$ is admissible.

Example 5.2. Let $R_{1}=\left\{(x, y) \in \mathbb{R}^{2}: y>x^{2}\right\}, R_{2}=\left\{(x, y) \in \mathbb{R}^{2}: y<-x^{2}\right\}$, and $\mathrm{R}_{3}=\mathbb{R}^{2} \backslash\left(\mathrm{R}_{1} \cup \mathrm{R}_{2}\right)$. Consider the $\mathrm{PC}^{1}$ map $\mathrm{f}: \mathbb{R}^{2} \rightarrow \mathbb{R}^{2}$ given by

$$
f(x, y)= \begin{cases}\left(x, 2 y-x^{2}\right) & \text { for }(x, y) \in R_{1} \\ \left(x, 2 y+x^{2}\right) & \text { for }(x, y) \in R_{2}, \\ (x, y) & \text { for }(x, y) \in R_{3} .\end{cases}
$$

One has that $\mathrm{f}^{\prime}((\mathrm{0}, 0), \cdot)$ is the identity and that the generalized Jacobian at the origin consists of matrices which have positive determinant. Hence, by Theorem 5.1. $\mathrm{f}$ is locally invertible about the origin.

In order to apply Theorem 5.1 above one needs to know when the linearization of a $\mathrm{PC}^{1}$ map (which is a continuous piecewise linear map) is invertible. This is what all the previous section is about. Criteria for the local invertibility of $\mathrm{PC}^{1}$ map will be deduced from Theorem 5.1 combined with the results of the previous section.

Let $f$ be an $\mathbb{R}^{k}$-valued $P C^{1}$ function in a sufficiently small ball $B\left(x_{0}, \rho\right) \subseteq \mathbb{R}^{k}$, and let $\mathcal{I}_{0}=\{1, \ldots, n\}$ be the active index set in $B\left(x_{0}, \rho\right)$. For each $i \in \mathcal{I}_{0}$ define

$$
S_{i}:=\left\{x \in B\left(x_{0}, \rho\right): f(x)=f_{i}(x)\right\} .
$$

Let $C_{1}, \ldots, C_{n}$ be the tangent cones (in the sense of Bouligand) at $x_{0}$ to the sectors $S_{1}, \ldots, S_{n}$. Assume that for $i, j \in\{1, \ldots, n\}$

$$
\mathrm{df}_{\mathrm{i}}\left(x_{0}\right) x=\mathrm{df}_{j}\left(x_{0}\right) x \text { for any } x \in C_{i} \cap C_{j},
$$

and define

$$
F(x)=\mathrm{df}_{\mathrm{i}}\left(\mathrm{x}_{0}\right) x \quad x \in \mathrm{C}_{i}, \mathrm{i}=1, \ldots, \mathrm{n}
$$

so that $\mathrm{F}$ is a continuous piecewise linear map (compare 6 ).

This section is concerned with local invertibility of such maps. We first consider arbitrary dimension.

Corollary 5.3. Let $\mathrm{f}$ and $\mathrm{F}$ be as in (5.1)-(5.2), with $\mathrm{F}$ nondegenerate at 0 . Assume also that there exists $\mathrm{p} \in \mathbb{R}^{\mathrm{k}}$ whose preimage under $\mathrm{F}, \mathrm{F}^{-1}(\mathrm{p})$, is a singleton that belongs to at most two of the cones $\mathrm{C}_{\mathrm{i}}$. Then $\mathrm{f}$ is a Lipschitzian homeomorphism in a sufficiently small neighborhood of $x_{0}$.

Proof. From Theorem 4.2 it follows that F is invertible. The assertion follows from Theorem 5.1.

The above Theorem 5.3 can be greatly simplified when the number of cones is $n=2$, in the sense that the assumption on the existence of the special point $p$ can be dropped altogether. In fact, in dimension $k=2$ this is true also for $k=3$ and, when $k=4$, one can replace it by merely requiring the convexity of the tangent cones to the sectors. 
Corollary 5.4. Let $\mathrm{f}$ and $\mathrm{F}$ be as in (5.1)-(5.2), with $\mathrm{F}$ nondegenerate at 0 and $\mathrm{n}=2$. Then $\mathrm{f}$ is a Lipschitzian homeomorphism in a sufficiently small neighborhood of $x_{0}$.

Proof. From Proposition 3.1 it follows that $\mathrm{F}$ is invertible. The assertion follows from Theorem 5.1

We finally consider dimension $k=2$ of the ambient space.

Corollary 5.5. Let $\mathrm{f}$ and $\mathrm{F}$ be as in (5.1) -(5.2), with $\mathrm{F}$ nondegenerate at 0 . We have that if either $\mathrm{n} \in\{1,2,3\}$ or $\mathrm{n}=4$ and all the cones $\mathrm{C}_{\mathrm{i}}$ 's are convex, then $\mathrm{f}$ is a Lipschitzian homeomorphism in a sufficiently small neighborhood of $x_{0}$.

Proof. Since F is nondegenerate then it is invertible by Theorem 4.5. Theorem 5.1. yields the assertion.

\section{REFERENCES}

[1] Andrei A. Agrachev and Yuri L. Sachkov. Control Theory from the Geometric Viewpoint. Springer-Verlag, 2004.

[2] Andrei A. Agrachev, Gianna Stefani, and PierLuigi Zezza. Strong optimality for a bang-bang trajectory. SIAM J. Control Optimization, 41(4):991-1014, 2002.

[3] Pierluigi Benevieri, Massimo Furi, Maria Patrizia Pera, and Marco Spadini. An introduction to topological degree in euclidean spaces. Technical Report memoria n. 42, Gennaio 2003, Università di Firenze, Dipartimento di Matematica Applicata, 2003.

[4] F.H. Clarke. Optimization and nonsmooth analysis. Unrev. reprinting of the orig., publ. 1983 by Wiley. Montréal: Centre de Recherches Mathématiques, Université de Montréal. , 1989.

[5] Klaus Deimling. Nonlinear Functional Analysis. Springer-Verlag, Berlin, 1985.

[6] Ludwig Kuntz and Stefan Scholtes. Structural analysis of nonsmooth mappings, inverse functions and metric projections. Journal of Mathematical Analysis and Applications, 188:346386, 1994.

[7] Lloyd N.G., Degree Theory, Cambridge Tracts in Math. 73, Cambridge University Press, Cambridge, 1978.

[8] John Milnor. Topology from the Differentiable Viewpoint. The University Press of Virginia, 1965.

[9] Jong-Shi Pang and Daniel Ralph. Piecewise smoothness, local invertibility, and parametric analysis of normal maps. Mathematics of Operations Research, 21(2):401-426, 1996.

[10] Laura Poggiolini and Marco Spadini. Sufficient optimality conditions for a bang-bang trajectory in a Bolza Problem, chapter In Mathematical Control Theory and Finance, Sarychev, A.; Shiryaev, A.; Guerra, M.; Grossinho, M.d.R. (Eds.), pages 337-358. Springer, 2008.

[11] Laura Poggiolini and Marco Spadini. Bang-bang trajectories with a double switching time: sufficient strong local optimality conditions, 2010.

[12] Laura Poggiolini and Gianna Stefani. State-local optimality of a bang-bang trajectory: a Hamiltonian approach. Systems \& Control Letters, 53:269-279, 2004.

(L. Poggiolini, M. Spadini) Dipartimento di Sistemi e Informatica, Università di Firenze, Via Santa Marta 3, 50139 Firenze, Italy 